\title{
Modal decomposition technique for multimode fibers
}

\author{
Duc Minh Nguyen, ${ }^{1, \star}$ Stéphane Blin, ${ }^{2}$ Thanh Nam Nguyen, ${ }^{1}$ Sy Dat Le, ${ }^{1}$ \\ Laurent Provino, ${ }^{3}$ Monique Thual, ${ }^{1}$ and Thierry Chartier ${ }^{1}$ \\ 'Université Européenne de Bretagne, France/CNRS (UMR6082) Foton, 6 rue de Kerampont 22300 Lannion, France \\ ${ }^{2}$ Institut d'Electronique du Sud, UMR 5214 CNRS, Université Montpellier 2, 34095 Montpellier, Cedex 5, France \\ ${ }^{3}$ Perfos, 11 rue Louis de Broglie, 22300 Lannion, France \\ ${ }^{*}$ Corresponding author: dnguyen@enssat.fr
}

Received 19 July 2011; revised 21 November 2011; accepted 23 November 2011;

posted 23 November 2011 (Doc. ID 151385); published 26 January 2012

\begin{abstract}
We propose a new solution for modal decomposition in multimode fibers, based on a spectral and spatial imaging technique. The appearance of spurious modes in the spectral and spatial processing of the images at the output of the fiber under test when it has more than two modes is demonstrated theoretically. The new method, which allows us to identify spurious modes, is more accurate, simpler, and faster than previously reported methods. For demonstration, measurements in a standard step-index multimode fiber and a small-core microstructured fiber are carried out successfully. @ 2012 Optical Society of America

OCIS codes: $\quad 060.2270,060.2320$.
\end{abstract}

\section{Introduction}

In the last decade, the advent of highly nonlinear microstructured fibers $[\underline{1}, \underline{2}]$ offers all-new possibilities in all-optical signal processing, such as data regeneration, time-division demultiplexing, or wavelength conversion. These applications are often based on the third-order nonlinear optical effects, which are limited by multimode propagation. However, singlemode propagation is not guaranteed in some microstructured fibers, either due to fiber design or imperfections in the fabrication process. On the other hand, multimode fibers are used in many applications, such as high powered lasers, to reduce optical nonlinear effects due to their large mode-areas or in sensing applications for utilizing modal interferences. Therefore, modal analysis of multimode fibers is necessary in order to identify the existing modes and to determine the power distribution within this set of modes. Several methods for a full modal decomposition have been proposed. With these methods, the transverse modes propagating in the fiber are

$1559-128 \mathrm{X} / 12 / 040450-07 \$ 15.00 / 0$

(C) 2012 Optical Society of America identified, as well as relative optical power, spatially resolved transverse amplitude, and phase of the electrical field [3-6]. The method proposed by Shapira et al. [3] determines experimentally the mode profiles using an algorithm based on the observation of near-field and far-field images at the waveguide output. The disadvantages, however, are the complexity of the algorithm and the need to acquire two images of near-field and far-field. A more direct measurement of the higher-order modes (HOMs) was made by using a bulk-optic Fabry-Perot resonator, but careful alignment of the bulk optics and a relatively large fraction of power $(>3 \%)$ in the HOMs are necessary [4]. Finally, methods described in [5,6] using differential delay measurements effectively discriminate transverse modes and measure relative optical power of each mode, but transverse mode amplitude profiles are not extracted. More recently, a new method has been introduced by Nicholson et al. $[\underline{7}, \underline{8}]$, namely the $S^{2}$ imaging, for spatially and spectrally resolved imaging of mode content in fibers. The technique is sensitive to HOMs and allows determination of accurate values of their relative optical power. However, the spatial solution is limited by diffraction limits, and only large-mode-area fibers can 
be characterized. The measurement is also time consuming due to the two-dimensional scanning of the mode at every position $(x, y)$ of the output surface of the large-core fiber, and it is limited to the case when the power of HOMs is small enough, in comparison to the fundamental mode, to be able to ignore interferences between two HOMs. In this paper, we demonstrate theoretically that spurious modes always appear in the $S^{2}$ imaging technique when the fiber under test has more than two modes. A new technique for modal decomposition whose algorithm is developed to the general case in which interferences between HOMs are taken into account is proposed. The measurement setup is improved as the twodimensional spatial scanning in the $S^{2}$ imaging technique is replaced by a unidimensional scanning of the wavelength. The characterization process is thus much faster, the method is not limited to large-modearea fibers, optical alignments are straightforward, and there is no moving part, since only near-field images are observed. The paper is organized as follows. Section 2 discusses spurious modes in the $S^{2}$ imaging method. Section $\underline{3}$ describes the principle of the new method. Experimental demonstration with a standard step-index multimode fiber and a small-core microstructured fiber is presented in Section $\underline{4}$.

\section{Spurious Modes in $\mathbf{S}^{2}$ Imaging Method}

Measurement based on interference effects is well known in optical systems, either due to its sensitivity or its accuracy. The $S^{2}$ imaging technique [7,8], which is based on the processing of the spectrally and spatially resolved interference images at the output of the fiber under test, is therefore a useful method for full modal decomposition. Spectral interference observed in the near-field images is caused by the group-delay difference between the HOMs and the fundamental mode propagating in the fiber. Figure 1 (a) presents an example of a typical optical spectrum measured at an arbitrary point $(x, y)$, where interferences between several transverse modes are locally observed. These interferences lead to several different mode beats at different time delays in the Fourier transform

of the spectrum, as shown in Fig. 1(b). If the fiber supports two transverse modes, there is only one spectral interference between the fundamental mode and the HOM. A peak in the Fourier transform of the optical spectrum obtained from each spatial point in the near-field image is then observed. In a more general case when the fiber has more than two modes, the $S^{2}$ imaging technique assumes that the power of HOMs is weak compared to the power of the primary $\mathrm{LP}_{01}$ mode, and thus interference between two different HOMs is considered negligible. This means that if the fiber supports $n$ HOMs, there will be $n$ different beat frequencies visible, corresponding to interferences between the primary $\mathrm{LP}_{01}$ mode and different HOMs. However, the condition that the HOMs are weak compared to the fundamental mode is ambiguous. We will demonstrate in this section that even when the relative power between the HOMs and the $\mathrm{LP}_{01}$ is low, the interference between the HOMs cannot be ignored. Let us suppose that the fiber has $n \mathrm{HOM}$ s and that their amplitudes $E_{i}$ are related to the amplitude $E_{0}$ of the fundamental mode $\mathrm{LP}_{01}$ by a constant $\alpha_{i}$ such that

$$
E_{i}(x, y, w)=\alpha_{i}(x, y, w) E_{0}(x, y, w) \exp \left(i w \tau_{i}\right)
$$

where $\tau_{i}$ is the period of the beat frequency between the corresponding $\mathrm{HOM}$ and the $\mathrm{LP}_{01}$ caused by their relative group-delay difference. The spectral intensity caused by interference between all modes propagating in the fiber can be written as

$$
\begin{aligned}
I(x, y, w)= & I_{0}(x, y, w)\left[1+\sum_{j=1}^{n} \alpha_{j}(x, y, w) e^{i w \tau_{j}}\right] \\
& \times\left[1+\sum_{k=1}^{n} \alpha_{k}(x, y, x) e^{-i w \tau_{k}}\right] \\
= & I_{0}\left(1+\sum_{j=1}^{n} \alpha_{j}^{2}+\sum_{j=1}^{n} \alpha_{j}\left(e^{i w \tau_{j}}+e^{-i w \tau_{j}}\right)\right. \\
& \left.+\sum_{j>k}^{n} \alpha_{j} \alpha_{k}\left(e^{i w\left(\tau_{j}-\tau_{k}\right)}+e^{-i w\left(\tau_{j}-\tau_{k}\right.}\right)\right),
\end{aligned}
$$
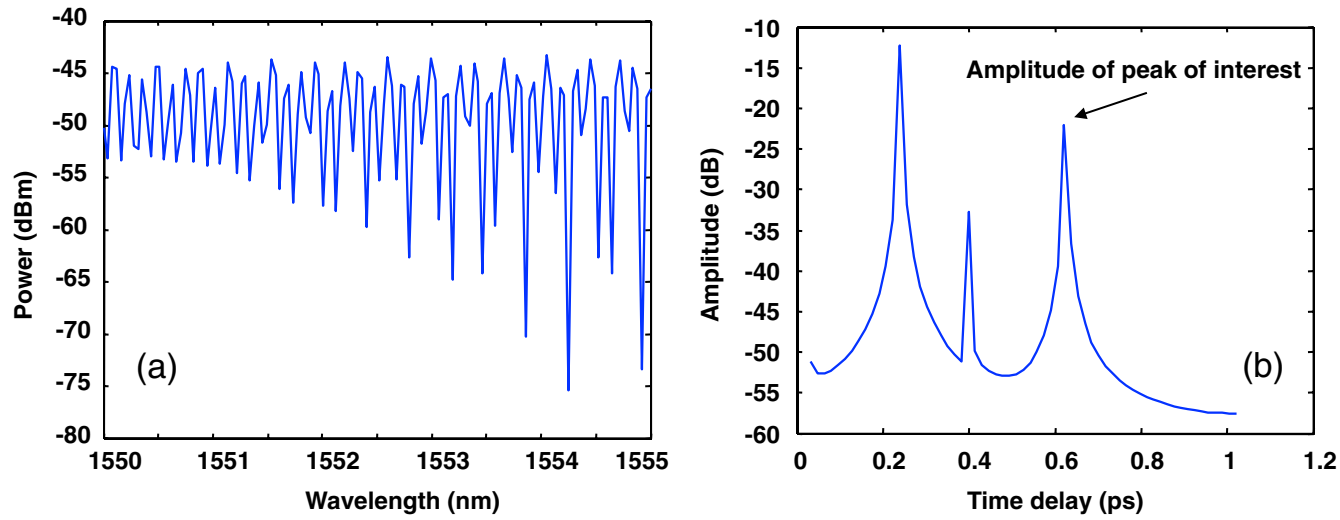

Fig. 1. (Color online) (a) Typical spectrum measured at an arbitrary $(x, y)$ point and (b) its Fourier transform showing multiple beat frequencies. 
with $I_{i}=\left|E_{i}\right|^{2}$. The Fourier transform of the spectral intensity is then

$$
\begin{aligned}
B(x, y, \tau)= & {\left[1+\sum_{j=1}^{n} \alpha_{j}^{2}(x, y)\right] B_{0}(x, y, \tau) } \\
& +\sum_{j=1}^{n} \alpha_{j}(x, y)\left[B_{0}\left(x, y, \tau-\tau_{i}\right)+B_{0}\left(x, y, \tau+\tau_{i}\right)\right] \\
& +\sum_{j>k}^{n} \alpha_{j}(x, y) \alpha_{k}(x, y)\left[B_{0}\left(x, y, \tau-\tau_{i}+\tau_{k}\right)\right. \\
& \left.+B_{0}\left(x, y, \tau+\tau_{i}-\tau_{k}\right)\right]
\end{aligned}
$$

where $B_{0}(x, y, \tau)=F\left\{I_{0}(x, y, w)\right\}$ is the Fourier transform of the optical spectrum of the fundamental mode. The second term in the right hand side of Eq. (3) is caused by the interference between the HOMs and the $\mathrm{LP}_{01}$ mode. It is also responsible for the appearance of $n$ peaks at time delay $\tau_{j}$ in the spatial-dependent Fourier transform of the optical spectrum. The ratio $f_{j}(x, y)$ of these peaks, which is defined as the amplitude of the Fourier transform of the spectral intensity at the corresponding group-delay difference divided by the amplitude at group-delay zero, can be then written as

$$
f_{j}(x, y)=\frac{B\left(x, y, \tau=\tau_{j}\right)}{B(x, y, \tau=0)}=\frac{\alpha_{j}(x, y)}{1+\sum_{j=1}^{n} \alpha_{j}^{2}(x, y)} .
$$

The last term in the right hand side of Eq. (3) comes from interferences between two HOMs. This term leads to additional $n ! /(2(n-2) !)=C_{n}^{2}$ peaks at time delay $\left|\tau_{j}-\tau_{k}\right|$ in the Fourier transform, in which their ratio $f_{j k}$ can be calculated from

$$
f_{j k}(x, y)=\frac{B\left(x, y, \tau=\tau_{j}-\tau_{k}\right)}{B(x, y, \tau=0)}=\frac{\alpha_{j}(x, y) \alpha_{k}(x, y)}{1+\sum_{j=1}^{n} \alpha_{j}^{2}(x, y)} .
$$

For simplicity, we call the peaks corresponding to interferences between the $\mathrm{LP}_{01}$ and the HOMs peaks
$P_{j}$ and the peaks corresponding to interference between two HOMs peaks $P_{j k}$. When the fiber has only one HOM or the relative power of the HOMs to the $\mathrm{LP}_{01}$ is very low, we return to the case explained in the $S^{2}$ imaging method [7], in which Eqs. (4) and (ㅁ) become

$$
\begin{gathered}
f_{j}(x, y)=\frac{\alpha_{j}(x, y)}{1+\alpha_{j}^{2}(x, y)}, \\
f_{j k}(x, y) \approx 0 .
\end{gathered}
$$

This means that peaks $P_{j k}$ vanish and only $n$ peaks $P_{j}$ are observed in the Fourier transform. Mode image and relative power of each HOM, as in the principle of the $S^{2}$ imaging method, are then extracted separately and independently from each peak $P_{j}$ from Eq. (6). In a general case when interferences between two $\overline{\mathrm{H}} \mathrm{OMs}$ are taken into account, $n$ peaks $P_{j}$ and $C_{n}^{2}$ peaks $P_{j k}$ are observed. Only modes extracted from $n$ peaks $P_{j}$ are real HOMs and modes extracted from the peaks $P_{j k}$ are spurious modes. It is also impossible to determine spatial intensity profile and relative power of HOMs independently from Eq. (6) instead of using Eq. (4). Moreover, the condition that the relative power of HOMs is low to ignore interferences between two HOMs is ambiguous. Interference between two HOMs $\mathrm{LP}_{11}$ and $\mathrm{LP}_{02}$ is discovered in the Fourier transform as shown in Fig. 2(a) even when their relative power to the primary mode $\mathrm{LP}_{01}$ is low, $-20 \mathrm{~dB}$ and $-30 \mathrm{~dB}$, respectively. As shown on this spectra, peaks are easily identified and accuracy of mode retrieval relies directly on the ratio between these peaks and the fast Fourier transform (FFT) background. In this figure, in addition to two real peaks $P_{1}, P_{2}$ at time delay $\tau_{1}, \tau_{2}$ corresponding to two HOMs $\mathrm{LP}_{11}$ and $\mathrm{LP}_{02}$, the spurious peak $P_{12}$ corresponding to interference between the $\mathrm{LP}_{11}$ and the $\mathrm{LP}_{02}$ at time delay $\tau_{2}-\tau_{1}$ is found. Figure $2(\mathrm{~b})$ is another example of the Fourier transform in the case of
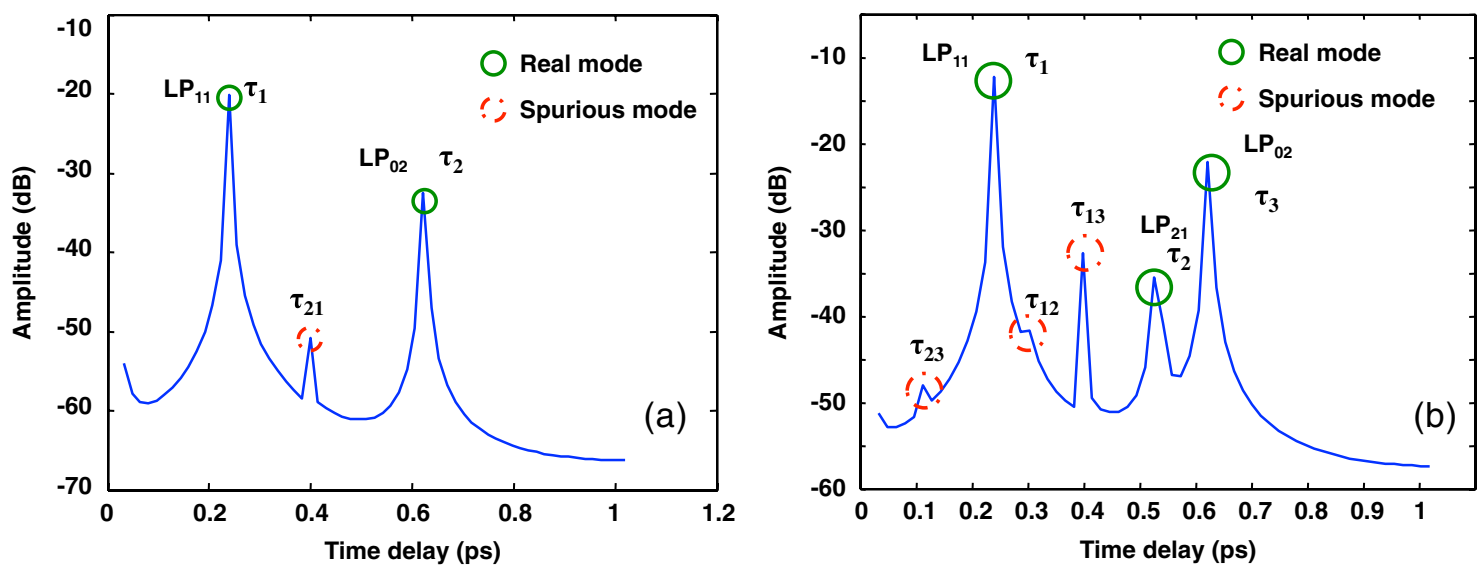

Fig. 2. (Color online) The Fourier transform when (a) the fiber supports two HOMs $\mathrm{LP}_{11}$ and $\mathrm{LP}_{02}$ and (b) when three HOMs are supported: $\mathrm{LP}_{11}, \mathrm{LP}_{21}$, and $\mathrm{LP}_{02}$. 
a fiber that supports three transverse HOMs $\mathrm{LP}_{11}$, $\mathrm{LP}_{02}$, and $\mathrm{LP}_{21}$ at relative powers of $-12 \mathrm{~dB},-21 \mathrm{~dB}$, and $-33 \mathrm{~dB}$, respectively. Three spurious peaks corresponding to mutual interferences between these HOMs are observed. Modes extracted from spurious peaks $P_{j k}$ are spurious modes that result from intrinsic properties of the wave propagation in the fiber. Besides, there is another kind of spurious mode that comes from extrinsic elements such as the instability of the measurement or the laser source. Since the Fourier transform reflects all phenomena causing interference at the output spectra, any oscillation in the measurement will lead to a spurious peak in the Fourier transform. In summary, the problem of spurious modes is really a challenge in the $S^{2}$ imaging method that can reduce the accuracy of the measurement, not only in the number of HOMs but also in their relative optical power. A full solution in the general case is therefore necessary.

\section{Modal Decomposition Technique}

In this section, we propose a new technique for full modal decomposition that is an alternative of the $S^{2}$ imaging method but with much improvement both in the calculated algorithms and the measurement setup. The algorithm used to retrieve modes in the $S^{2}$ imaging technique is based on the hypothesis that one mode (usually the fundamental one) is much more powerful than the others, and thus neglects the possible interferences between other modes. These interferences are misinterpreted by the algorithm, which leads to the apparition of spurious modes at the algorithm output. We present an alternative to this algorithm that is not limited to the case of a dominant mode, and provide a solution to identify and then eliminate these spurious modes, thus offering artifact-free analysis of the measurement. Moreover, replacement of a broadband laser source with a tunable laser source helps the new technique to have only one-dimensional scanning, instead of twodimensional scanning in the previous method. The measure is therefore faster, simpler, and more stable.

The experimental setup is presented in Fig. 3 . The implementation is simple, although it is the first time, to the best of our knowledge, that such a setup is used both to discriminate transverse modes and to determine their spatial profiles. Light from a tunable source is coupled to the fiber under test. Drawbacks of the $S^{2}$ imaging method due to a two-spatial dimensional scanning, which is necessary to measure the magnified image of the fiber end with a single-mode

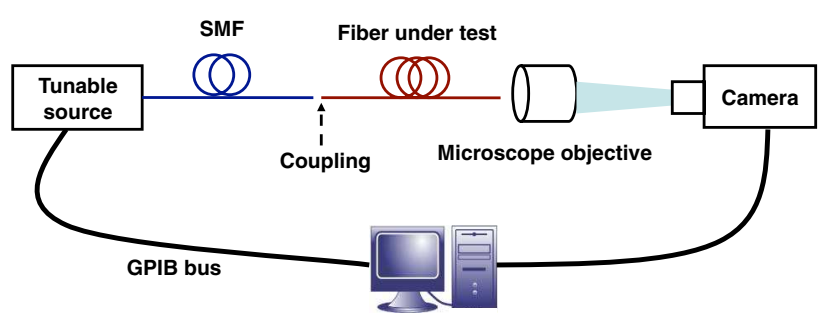

Fig. 3. (Color online) Experimental setup for the new technique. fiber, are alleviated by simply scanning the wavelength of the tunable source and observing the magnified near-field image of the fiber end using a camera, as presented in Fig. 3 . As transverse modes do not propagate at the same speed in the fiber, we observe an interference pattern at the fiber output (as previously described in Eq. (2)). At every position $(x, y)$ of this pattern, the interference state depends on modes amplitudes, but also on relative propagation phases (which thus depend on wavelength). As explained in the previous section, the interference states at every position evolve periodically as the wavelength is increased. The magnified near-field image of the fiber output is recorded every time the wavelength of the source is changed. Transmission spectra at each position $(x, y)$ as shown in Fig. 1(a) are then extracted from the set of images, acquired at each wavelength of the source. The integrated Fourier transform of these optical spectra (cf. Figure 1(b)) is then calculated. Maximum peaks in the Fourier transform will be used to retrieve all the existing modes. However, spurious peaks that correspond to interferences between two HOMs or an oscillating instability of the measurement must be excluded first. An exclusion technique consisting of two steps is implemented to solve this problem.

The first step is to remove spurious peaks that are not caused by intrinsic properties of the wave propagation in the fiber. Since time delays of the peaks related to interferences between different modes propagating in the fiber are always proportional to the fiber length, while this does not occur for the spurious peaks that do not come from intrinsic properties of the fiber, we can eliminate spurious peaks by measuring the fiber with two different lengths. The peak whose time delay remains when the fiber length changes is a spurious peak and will be eliminated.

The last step is to remove spurious peaks resulting from interferences between two HOMs. Since the power of HOMs is normally much smaller than that of the fundamental mode, the coefficient $\alpha_{j}$ in Eqs. (4) and (ㅁ) is smaller than 1 . We can also see from these two equations that the ratio $f_{j k}$ of the peak $P_{j k}$ corresponding to the interference between the mode $P_{j}$ and the mode $P_{k}$ is always smaller than the ratio $f_{j}$ and $f_{k}$ of the peaks $P_{j}$ and $P_{k}$. The two highest peaks $P_{1}$ and $P_{2}$ at time delays $\tau_{1}$ and $\tau_{2}$ in the Fourier transform are then real peaks due to this property. The first spurious peak $P_{12}$ is then determined at the position $\left|\tau_{2}-\tau_{1}\right|$. Amplitude of this spurious peak is also larger than other spurious peaks because it corresponds to the interference of the two largest HOMs. All other peaks whose amplitudes are higher than the one of this peak are therefore real peaks. Spurious peaks that come from interferences between these real peaks can be then determined according to their differences in time delays. We call these spurious peaks the $P_{12-s}$. The highest peak $P_{3}$ that is lower than the peak $P_{12}$ and is not one of the peaks $P_{12-s}$ is also a real peak. All remaining peaks that are higher than $P_{3}$ are real 
peaks, and spurious modes corresponding to interferences between these real peaks can be then calculated. The same procedure is implemented until all peaks are classified.

In summary, using the exclusion technique, we can determine all real peaks $P_{j}$ and eliminate other spurious peaks. All the existing modes are retrieved using the ratio $f(x, y)$ of these peaks. From Eq. ( $\underline{4})$, the coefficient is related to $f_{j}(x, y)$ by

$$
\alpha_{j}(x, y)=\frac{f_{j}\left(1-\sqrt{1-4 \sum_{1}^{n} f_{k}^{2}}\right)}{2 \sum_{1}^{n} f_{k}^{2}} .
$$

The intensities of HOMs are then given by

$$
I_{j}(x, y)=I_{T}(x, y) \frac{\alpha_{j}^{2}(x, y)}{1+\sum_{k=1}^{n} \alpha_{k}^{2}}
$$

where $I_{T}(x, y)$ is the integrated optical spectrum at a given $(x, y)$ point. Relative optical power and spatially resolved transverse amplitude can be calculated from Eq. (9).

Moreover, with the new measurement setup, only one-dimensional scanning required on the wavelength is done. In the $S^{2}$ technique, the two-spatial dimensional scanning requires careful optical alignments at the fiber under test output, which is time consuming and can require multiple hours of scanning to achieve a sufficient solution. These alignments are much simpler in our technique, as transverse/angular alignments can be approximate. The new technique is therefore simpler and not time consuming. It takes several minutes for a measurement.

Accuracies in reproducing modal images or relative modal powers are improved due to the faster nature of the experiment and new algorithms in which interferences between two HOMs are taken into account.

As the previously published technique is slow, modal content could change during the scanning, thus conducting to erroneous mode images (and thus erroneous relative modal power). Note that the more modes are propagating in the fiber, the faster the interference figure at the fiber output changes. Therefore, the faster technique we propose allows for more accurate imaging of a multimode fiber supporting few modes, or allows characterizing a fiber supporting more modes.

The accuracy of the measurement is also improved by using the new algorithms of the new technique in which the interferences between two HOMs are taken into account. In the case of a multimode fiber supporting three HOMs, which is expressed in Section 2 and in Fig. 2(b), relative powers between HOMs and the fundamental mode calculated by the $S^{2}$ technique, in which interferences between two HOMs are ignored, and the values calculated by the new algorithms are compared in Table 1 . A better
Table 1. Relative Power Calculated by the Previously Published Algorithms and the New Algorithms

\begin{tabular}{llll}
\hline & \multicolumn{1}{c}{$\mathrm{LP}_{1} 1$} & \multicolumn{1}{c}{$\mathrm{LP}_{0} 2$} & \multicolumn{1}{c}{$\mathrm{LP}_{1} 2$} \\
\hline Given values & $-12 \mathrm{~dB}$ & $-21 \mathrm{~dB}$ & $-33 \mathrm{~dB}$ \\
$S^{2}$ technique & $-12.1 \mathrm{~dB}$ & $-22.2 \mathrm{~dB}$ & $-34.3 \mathrm{~dB}$ \\
New technique & $-12.08 \mathrm{~dB}$ & $-21.7 \mathrm{~dB}$ & $-33.16 \mathrm{~dB}$ \\
\hline
\end{tabular}

accuracy is obtained with the new technique compared to the $S^{2}$ technique.

\section{Experimental Results}

For the experimental demonstration of this technique, we used the experimental setup presented in Fig. 3 with a commercial C-band external-cavity laser. The laser wavelength is swept from $1540 \mathrm{~nm}$ to $1550.24 \mathrm{~nm}$ with 1024 steps. Light propagates in a standard single-mode fiber whose mode field diameter is about $10.4 \mu \mathrm{m}$. This fiber is butt-coupled to the fiber under test. A microscope objective with a magnification of 80 is used to image the near-field at the fiber output. Images are captured using a camera, which returns an 8 bit $200 \times 200$ points image. The experimental data acquisition was done in a few minutes, thus offering great environment stability. Data processing also requires a few minutes and returns mode intensity profiles, along with their relative optical power. Two fibers are tested, a standard step-index multimode fiber and a small-core microstructured fiber, respectively.

\section{A. Standard Step-Index Multimode Fiber}

We mesure $0.5 \mathrm{~m}$-long and $1 \mathrm{~m}$-long standard multimode fibers using the new technique. The core diameter of the fiber is $12 \mu \mathrm{m}$, and the core/cladding index difference is $9.810^{-1}$. This fiber should therefore support four LP modes: $\mathrm{LP}_{01}, \mathrm{LP}_{11}, \mathrm{LP}_{21}$, and $\mathrm{LP}_{02}$. The new technique takes several minutes for measurement. Figure $\underline{4(\mathrm{a})}$ presents the Fourier transform measured for the $0.5 \mathrm{~m}$-long fiber. Three peaks are found. However, the time delay of $4.18 \mathrm{ps}$ of the last peak remains unchanged when the fiber length is changed to $1 \mathrm{~m}$. As explained in the principle of the technique, the last peak is a spurious peak. Expected mode profiles and their relative optical powers are then retrieved using the algorithm (8) and (9), as shown in Fig. 4(b). The fact that the $\mathrm{LP}_{21}^{-}$ was not observed in the measurement is not due to the principle technique. The technique allows us to characterize the modal content at the fiber output for a given configuration (optical injection, bending, etc.). However, the number of modes excited in our case is only three $\left(\mathrm{LP}_{01}, \mathrm{LP}_{11}\right.$, and $\left.\mathrm{LP}_{02}\right)$ due to the optical injection. It is then evident that the technique discovers only three modes. All four modes are extracted in another measurement for the same fiber but with another configuration of optical injection, as presented in Fig. 5(b). A good agreement with the theoretical modes in Fig. 5(a) confirms the method. To give more proof of the method, an effort to find the origin of the extrinsic spurious mode is 


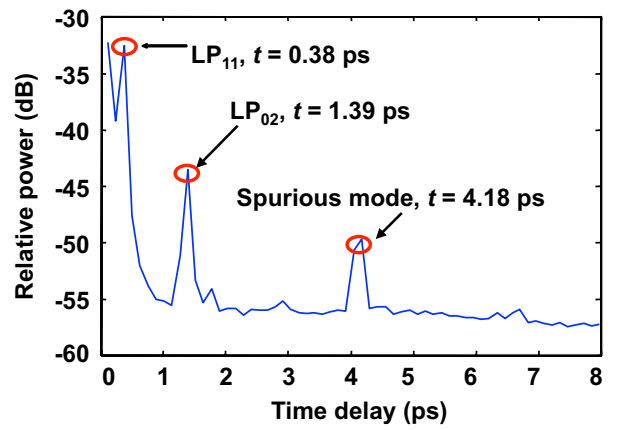

(a)

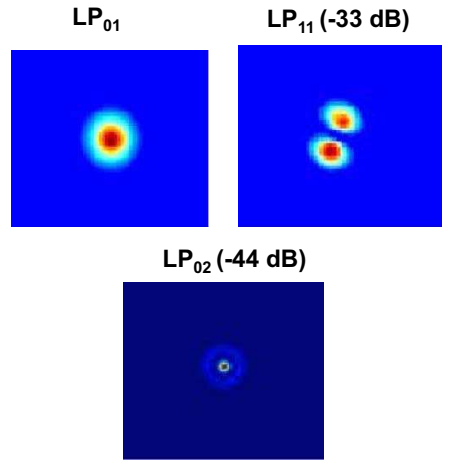

(b)

Fig. 4. (Color online) (a) The Fourier transform measured by the new technique for the standard multimode fiber and (b) the HOM profiles and their relative powers.

(a) Theory
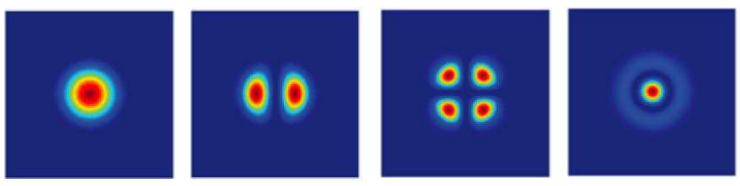

(b) Measure
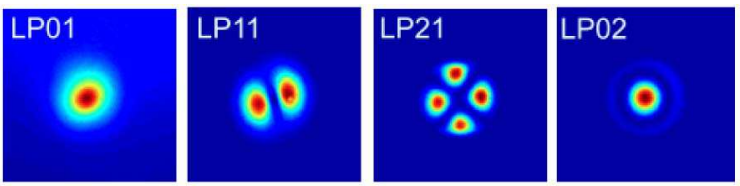

Fig. 5. (Color online) (a) Theoretical modes and (b) modes extracted by the technique in the new measurement for the same standard step-index fiber but with the new condition of the optical injection.

performed. In the principle, the power of the source must be fixed, while the laser wavelength is varied. However, by investigating the power of the laser source as a function of the wavelength, a small modulation of the source power as a function of the wavelength with a period $\Delta \lambda$ of $3.8 \mathrm{~nm}$ is found. This modulation leads to a peak in the Fourier transform with the corresponding time delay calculated from

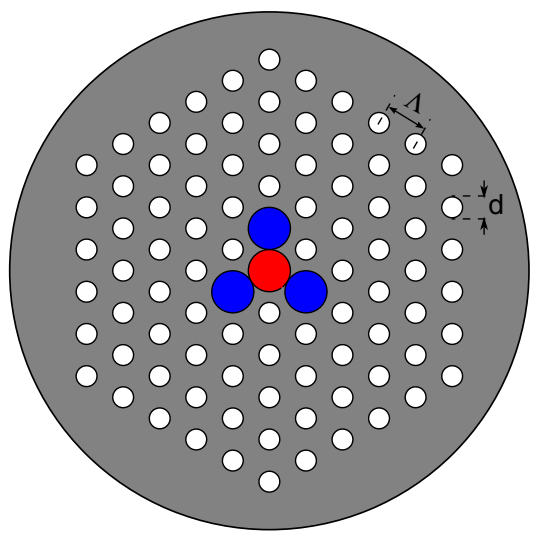

Fig. 6. (Color online) Transverse section of the photonic-crystal fiber. $\Lambda=1.5 \mu \mathrm{m}, d=0.75 \mu \mathrm{m}$. $\tau=2 \lambda^{2} /(c \Delta \lambda)=4.18 \mathrm{ps}$. This value is the same as the time delay of the spurious peak. Consequently, we can conclude that the spurious mode in the experiment results from the power instability of the source, and once again, the spurious mode, as well as the principle of the new technique, is verified.

\section{B. Microstructured Fiber}

The second fiber under test is a 1.5-m-long commercially available solid-core microstructured fiber whose fundamental mode has a $2.8-\mu \mathrm{m}$-averagediameter triangular shape. The structure of the fiber is sketched in Fig. 6. The triangular core region comprises a germanium-doped center element (red) surrounded by three fluorine-doped rods (blue) embedded in a standard triangular air/silica cladding structure with lower mean refractive index. The diameter of the doped elements equals the pitch. Experimental results are shown in Fig. 7. The fundamental mode and two HOMs are observed. Theoretical modes of the fiber were also calculated, and a good agreement was found with experimental results only if a transverse index inhomogeneity was included in the fiber structure. HOMs 1 and 2 would both have the same intensity profiles for a perfectly homogenous fiber. The main limitation of this experiment
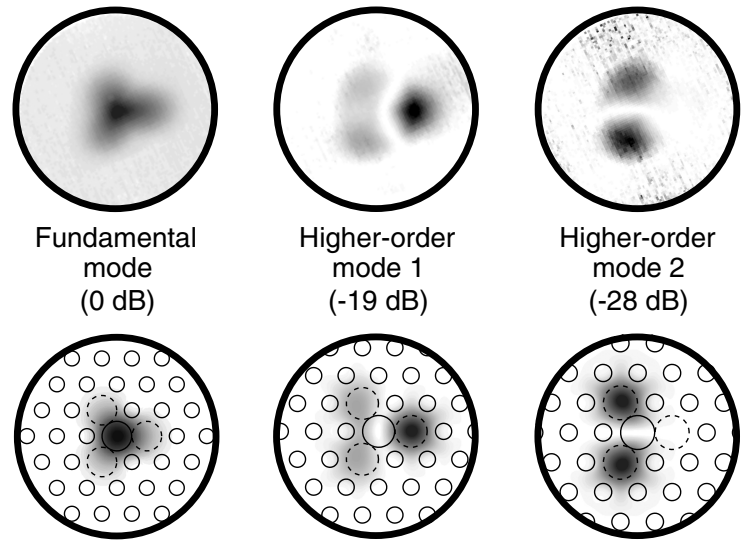

Fig. 7. Experimental (top) and numerical (bottom) intensity profiles of transverse modes for a small-core photonic-crystal optical fiber. 
is the need for a tunable laser source, which is not available around some wavelengths. Therefore, we replaced the tunable laser source with the combination of a superfluorescent source and a tunable optical fiber with a $0.27 \mu \mathrm{m}$ spectral width. Similar results were obtained, thus indicating that optical fibers can be characterized at some exotic wavelengths. Additionally, the cost of the experimental setup can be reduced.

\section{Conclusion}

A new method for modal analysis of optical fibers was proposed and demonstrated. This method alleviates most of the drawbacks of existing techniques. The algorithm of the method is developed to be able to take into account interferences between two HOMs. The method can thus eliminate spurious modes and offer a more accurate measurement. The characterization process is improved by replacing a two-dimensional scanning in the $S^{2}$ imaging technique with unidimensional scanning. This setup is simple and suitable to any optical-fiber core size and not only limited to large-mode-area fibers. A standard step-index multimode fiber and a small-core microstructured fiber have been used to demonstrate the method.
Authors are thankful to Thierry Robin and Benoit Cadier (iXfiber, Lannion, France) for providing the step-index fiber.

\section{References}

1. D. M. Nguyen, S. D. Le, K. Lengle, D. Mechin, M. Thual, T. Chartier, Q. Coulombier, J. Troles, L. Bramerie, and L. Brilland, "Visual system-response functions and estimating reflectance," IEEE Photon. Technol. Lett. 22, 1844-1846 (2010).

2. B. J. Eggleton, B. Luther-Davies, and K. Richardson, "Chalcogenide photonics," Nat. Photon. 5, 141-148 (2011).

3. O. Shapira, A. F. Abouraddy, J. D. Joannopoulos, and Y. Fink, "Complete modal decomposition for optical waveguides," Phys. Rev. Lett. 94, 143902-143905 (2005).

4. N. Andermahr, T. Theeg, and C. Fallnich, "Novel approach for polarization-sensitive characterization of transversal modes in few-mode optical fibers," Appl. Phys. B 91, 353-357 (2008).

5. T.-J. Ahn and D. Y. Kim, "High-resolution differential mode delay measurement for a multimode optics fiber using a modified optical frequency domain reflectometer," Opt. Express 13, 8256-8262 (2005).

6. S. Ring, D. Menashe, U. Levy, S. Steinblatt, Y. Danziger, and M. Tur, "Characterization in mode coupling in few-mode fibers using optical low-coherence reflectometry," in Optical Fiber Communication Conference (2008), paper OWO5.

7. J. W. Nicholson, A. D. Yablon, S. Ramachandran, and S. Ghalmi, "Spatially and spectrally resolved imaging of modal content in large-mode area fibers," Opt. Express 16, 7233-7243 (2008).

8. J. W. Nicholson, A. D. Yablon, J. M. Fini, and M. D. Mermelstein, "Measuring the modal content of large-mode-area fibers," IEEE J. Sel. Top. Quantum Electron. 15, 61-70 (2009). 